\title{
Duplication of the Extracranial Internal Carotid Artery Analyzed by MRI
}

\author{
Max Scheffler, Victor Cuvinciuc, Karl-Olof Lovblad, Maria Isabel Vargas
}

Can J Neurol Sci. 2013; 40: 423-425

A 68-year-old female presenting with gait instability and vertigo for six months was referred to our institution for cranial magnetic resonance imaging (MRI) to rule out acoustic neuroma or stroke. Her past medical history was noteworthy for hypertension, hypercholesterolemia, obesity, and diabetes. The neurological examination revealed no focal neurological deficit. Magnetic resonance imaging of the head and the vessels of the neck was performed on a 1.5 Tesla machine (Magnetom Espree; Siemens, Germany) with acquisition sequences comprising axial T1 weighted imaging, axial T2 weighted imaging, coronal fluid attenuated inversion recovery (FLAIR), axial gradient echo T2, axial diffusion weighted imaging, axial three-dimensional (3D) time of flight MRI angiography, and coronal MRI angiography of the supraaortic vessels after injection of $23 \mathrm{ml}$ of paramagnetic gadolinium-based contrast media (Dotarem; Guerbet, Roissy, France). Multiplanar maximum intensity pixel and $3 \mathrm{D}$ reconstructions were performed.

The MRI revealed neither an anomaly of the patient's internal auditory canals, nor signs of recent stroke. However, particularly well seen on the angiographic $3 \mathrm{D}$ reconstructions, was a duplication of the right extracranial internal carotid artery (ICA) starting $1.8 \mathrm{~cm}$ above the common carotid bifurcation with extension to the base of the skull and the beginning of the petrous portion of this artery, where the two lumens reunited (Figures 1,2). The diameter of the two rounded lumens was equal, and the distance between them too great for a diagnosis of dissection to be entertained (Figure 2). The course of the duplicated ICA was within normal limits, the two branches parallel. Just caudal to their reunification, a small $3.5 \mathrm{~mm}$ aneurysm was seen arising from the more medial of the two branches (Figure 1).

Several variations may occur in carotid anatomy, in the course of the common carotid artery (CCA) and the ICA, as well as in the position of the carotid sinus ${ }^{1}$. Persistent embryologic arteries comprise the trigeminal artery, the hypoglossal artery, otic and proatlantal intersegmental arteries ${ }^{1}$. Duplication of the extracranial ICA is a rarely seen anatomic variant. Duplication must be differentiated from dissection as well as fenestration, the latter referring generally to a shorter segment. In the scientific literature, ICA duplication has been described by Killien et al in $1980^{2}$, and then by Chess et al in $1995^{3}$, and McEachen et al in 2008 , in association with other developmental anomalies ${ }^{4}$.

In the human embryo, vasculogenesis commences on day 17 in the yolk sac mesoderm, and on day 18 in the embryonic disc, where endothelial cell precursors form so-called "angioblastic cords" . These "angioblastic cords" coalesce to form a network of angioblastic plexuses, the base of the circulatory system of the embryo $^{5}$. On day 19 , vascular elements form in the cardiogenic

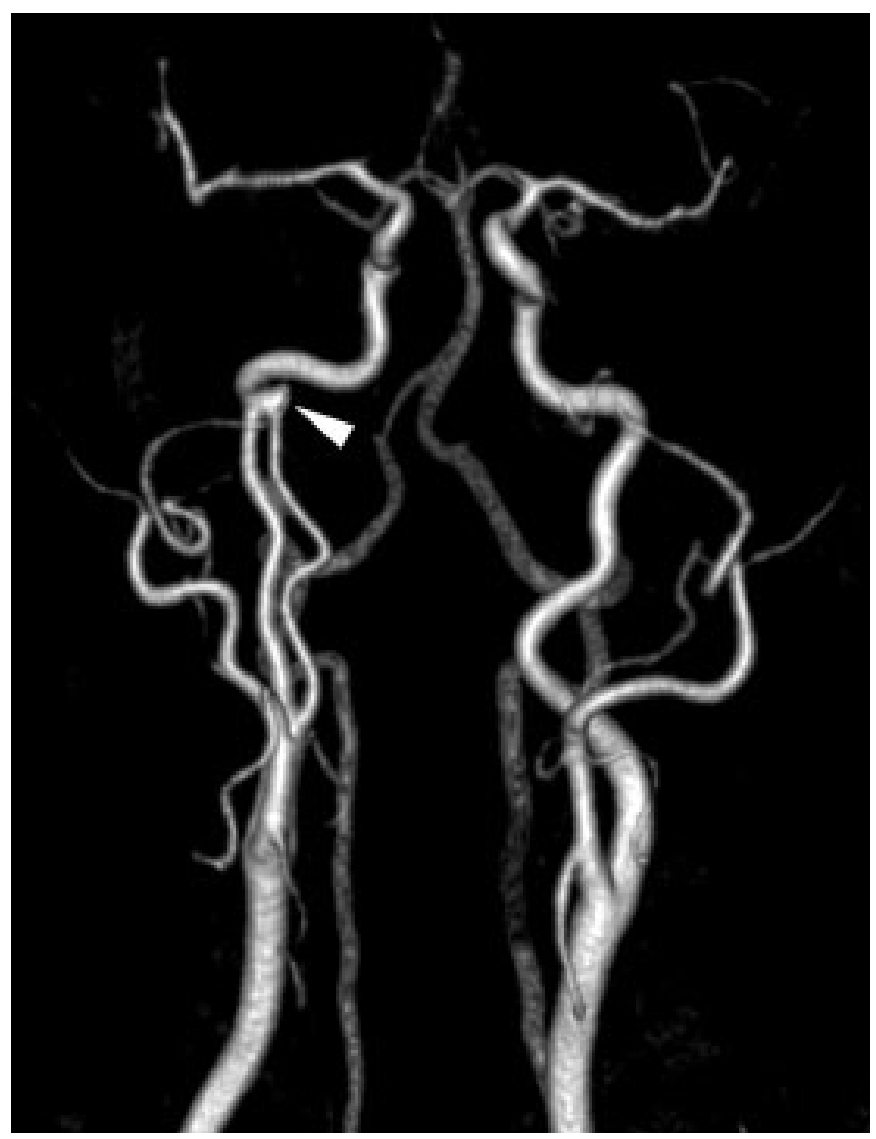

Figure 1: 3D-reconstructed gadolinium-enhanced T1-weighted echogradient angio-MRI of a 68-year-old woman showing right extracranial ICA duplication associated with a subcranial aneurysm (arrowhead).

region, the endocardial tubes. At the same time, paired dorsal aortae develop in the dorsal part of the embryo. The endocardial tubes form the primitive heart tube, with the truncus arteriosis located at its outflow end 5 .

From the Departments of Radiology (MS), Neuroradiology (VC, KOL, MIV), University of Geneva and Geneva University Hospitals, Geneva, Switzerland. Received September 12, 2012. Final Revisions Submitted December 11, 2012. Correspondence to: Maria Isabel Vargas, Service de neuroradiologie, Hôpitaux Universitaires et Université de Genève, 4 Gabrielle-Perret-Gentil, 1211 Genève 14, Switzerland. Email: maria.i.vargas@hcuge.ch. 


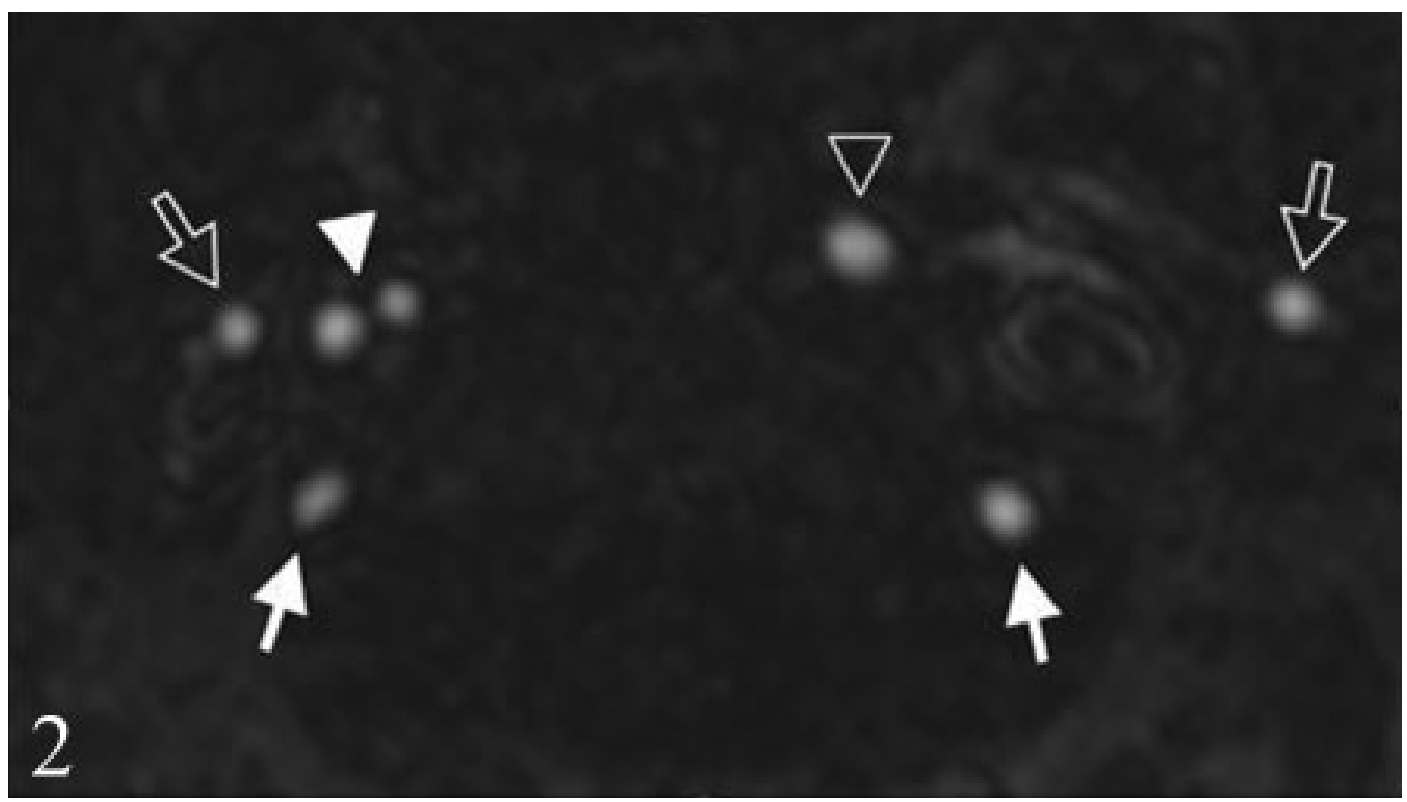

Figure 2: Transverse reconstruction of gadolinium-enhanced T1-weighted echo-gradient angio-MRI showing right cervical ICA duplication (white arrowhead). Note left normal cervical ICA (black arrowhead), external carotid arteries (black arrows), and vertebral arteries (white arrows).

The great vessels of the upper thorax and the neck in humans arise from the branchial arch system, a remnant of the branchial respiratory system in agnathan fish. During the fourth and fifth weeks of gestation (4-5 mm embryonal stage), five branchial arches develop on each side of the pharynx, out of mesenchymal condensations with mesodermal and endodermal components, in a craniocaudad sequence ${ }^{5}$. Initially, these arches contain multiple arterial channels, the aforementioned angioplastic plexuses, connecting (anastomosing) the aortic sac extension of the truncus arteriosus (ventral aorta) with the dorsal aorta. Subsequently, with ongoing angiogenesis, aortic arch arteries 1, $2,3,4$, and 6 form in remodeling processes, arch 5 remaining rudimentary or non-existant in human development ${ }^{1}$. The entirety of these arteries conveys, initially, oxygenated blood to the paired dorsal aorta.

The first two arches form between days 22 and 24 and involute in humans without significant remains apart from stapedian and maxillary arteries ${ }^{3}$. Arches 3, 4, and 6 develop between days 26 and 29. On either side, the proximal, extracranial portion of the ICA is a derivative of the proximal portion of the third aortic arch, also known as "carotid arch", whereas the intracranial portion of the ICA is formed by the distal portion of the third aortic arch and the ipsilateral cranial dorsal aorta. By 35 days of embryonic age, the segments connecting arches 3 and 4 of the dorsal aortae, the "trunci carotici", disappear by regression. The CCA also develop out of the third aortic arches ${ }^{5}$, with contribution of the ventral aortic $\mathrm{sac}^{1}$. Branches of the latter also form the external carotid arteries ${ }^{1}$. The left fourth aortic arch forms a part of the normal adult aortic arch and the sixth aortic arches finally develop into the pulmonary arteries and the ductus Botalli ${ }^{1}$.

Embryogenic vasculogenesis is thus a process comprising regression, remodeling, and fusion/anastomosis of primitive vessels or plexuses. Regression affects mainly the right side of the aortic arch system ${ }^{5}$. Most vascular malformations result from the failure of some primitive elements to undergo regression ${ }^{5}$.

In the case here described, the duplication seems to be due to a developmental abnormality of the right-sided third aortic arch, possibly in the form of an incomplete consolidation of the third arch's plexiform arterial channels described above into the normal vessels. In our case, the finding was associated with a small aneurysm of one of the two lumens, just below the skull base. It remains unclear whether this aneurysm existed from the outset, as an associated congenital anomaly, or whether it developed secondarily due to altered blood flow dynamics and perhaps an altered vascular media. To our best knowledge, this is the first case of an aneurysm described in association with extracranial ICA duplication.

Other vascular variants have been described in combination with aneurysms, such as fenestration of vertebral arteries and supraclinoid ICA fenestration. Several cases of middle cerebral artery duplication have been described with concomitant aneurysms $s^{6,7}$. Duplication of the extracranial ICA on the other hand has been reported in association with severe stenosis ${ }^{8}$, posing the question of an increased risk for the formation of atherosclerotic lesions.

In conclusion, we report a case of duplication of the extracranial ICA, a rare anatomic variant, usually incidentally discovered and requiring no particular treatment. Its importance lies in differentiating it from carotid dissection. It also needs to be inscribed in the patient's medical record in case of possible future neck or carotid surgery or angioplasty. Moreover, in our case an aneurysm was associated with the duplication, warranting follow-up imaging. 


\section{REFERENCES}

1. Dungan DH, Heisermann JE. The carotid artery. Embryology, normal anatomy, and physiology. Neuroimag Clin N Am. 1996; 4:789-99.

2. Killien FC, Wyler AR, Cromwell LD. Duplication of the internal carotid artery. Neuroradiology. 1980;19:101-2.

3. Chess MA, Barsotti JB, Chang JK, Ketonen LM, Westesson PL. Duplication of the extracranial internal carotid artery. Am J Neuroradiol. 1995;16:1545-7.

4. McEachen JC, Obrzut M, Bokhari SJ. A rare combination of carotid artery congenital abnormalities: understanding the embryology and clinical associations. Emerg Radiol. 2009;16:411-4.
5. Larsen WJ. Human embryology. 3rd ed. Philadelphia: Churchill Livingstone; 2001.

6. Kai Y, Hamada J, Morioka M, Yano S, Kudo M, Kuratsu J. Treatment of unruptured duplicated middle cerebral artery aneurysm: case report. Surg Neurol. 2006;65:190-3 .

7. Hori E, Kurosaki K, Matsumura N, et al. Multiple aneurysms arising from the origin of a duplication of the middle cerebral artery. J Clin Neurosci. 2005;12:812-5.

8. Kung DK, Lui W, Smoker WR, Hasan DM. Duplication of the internal carotid artery presenting with severe artherosclerotic stenosis. J Clin Neurosci. 2011;18:982-3. 